\title{
Integrating Support Persons into Diabetes Telemonitoring to Improve Self-Management and Medication Adherence
}

\author{
James E. Aikens, $P h D^{7}$, Ranak Trivedi, PhD ${ }^{2,3}$, David C. Aron, MD, MS ${ }^{4,5}$, and John D. Piette, MSc PhD 6,7 \\ 'Department of Family Medicine, University of Michigan, Ann Arbor, MI, USA; ${ }^{2}$ Center for Innovation to Implementation, VA Palo Alto Health Care \\ System, Palo Alto, CA, USA; ${ }^{3}$ Department of Psychiatry and Behavioral Sciences, Stanford University, Palo Alto, CA, USA; ${ }^{4}$ Department of Medicine, \\ Case Western Reserve University, Cleveland, OH, USA; ${ }^{5}$ Louis Stokes VA Cleveland Medical Center, Cleveland, OH, USA; ${ }^{6} \mathrm{Ann}$ Arbor Department of \\ Veterans Affairs Center for Clinical Management Research, Ann Arbor, MI, USA; ${ }^{7}$ Department of Health Behavior and Health Education, School of \\ Public Health, and Department of Internal Medicine, University of Michigan, Ann Arbor, MI, USA.
}

OBJECTIVE: The purpose of this study was to investigate the potential benefits for medication adherence of integrating a patient-selected support person into an automated diabetes telemonitoring and self-management program, and to determine whether these benefits vary by patients' baseline level of psychological distress.

STUDY DESIGN: The study was a quasi-experimental patient preference trial.

METHODS: The study included patients with type 2 diabetes who participated in three to six months of weekly automated telemonitoring via interactive voice response (IVR) calls, with the option of designating a supportive relative or friend to receive automated updates on the patient's health and self-management, along with guidance regarding potential patient assistance. We measured long-term medication adherence using the four-point Morisky Medication Adherence Scale (MMAS-4, possible range 0-4), weekly adherence with an IVR item, and psychological distress at baseline with the Mental Composite Summary (MCS) of the SF-12.

RESULTS: Of 98 initially nonadherent patients, $42 \%$ opted to involve a support person. Participants with a support person demonstrated significantly greater improvement in long-term adherence than those who participated alone (linear regression slopes: -1.17 vs. -0.57 , respectively, $p=0.001)$. Among distressed patients in particular, the odds of weekly nonadherence tended to decrease $25 \%$ per week for those with a support person ( $p=0.030$ ), yet remained high for those who participated alone $(p=0.820)$.

CONCLUSIONS: Despite their multiple challenges in illness self-management, patients with diabetes who are both nonadherent and psychologically distressed may benefit by the incorporation of a support person when they receive assistance via automated telemonitoring.

KEY WORDS: diabetes; telehealth; primary care; care management.

J Gen Intern Med 30(3):319-26

DOI: $10.1007 / \mathrm{s} 11606-014-3101-9$

(C) Society of General Internal Medicine 2014

Received July 22, 2014

Revised September 19, 2014

Accepted October 15, 2014

Published online November 25, 2014

\section{INTRODUCTION}

Poorly controlled blood glucose in type 2 diabetes increases the risk of chronic hyperglycemia, microvascular complications, and heart disease. ${ }^{1}$ Most patients are advised to perform numerous daily self-management behaviors, including medication regimens that vary in complexity. Regular adherence to antihyperglycemic medication is, by far, one of the best predictors of long-term glycemic control. ${ }^{2}$ Although there are many factors involved in medication adherence, clinician-patient interactions are often an effective intervention. $^{3}$ Factors that impact negatively on the number and quality of these interactions, however, include limited numbers of trained clinicians, clinician time constraints, and third-party reimbursement. ${ }^{4}$

Automated telemonitoring may help address these systemlevel limiters of effective diabetes self-management support. ${ }^{5-7}$ We have developed an automated telemonitoring service for diabetes (the "CarePartner Program") that uses interactive voice response (IVR) telemonitoring, in which patients respond to prerecorded queries using their telephone keypad. Based on patients' IVR responses, the system provides tailored selfmanagement messages and notifies the patient's clinical team about health and self-management problems that may require additional follow-up. Analyses of patients' program engagement and their reports during IVR calls indicate that the program provides valid and actionable feedback to clinicians, and may improve self-management, diabetes-related distress, functional status, and blood glucose stability. ${ }^{8-10}$

Another potentially helpful strategy for improving patient self-management is to empower a family member or close friend to be a more effective helper by providing them with information about diabetes self-management and the patient's status. While significant others often assist patients with managing their diabetes, they are at elevated risk for caregiver burnout and may not have the information they need to be fully effective. ${ }^{11,12}$ Moreover, many people with diabetes live alone and receive support from caregivers living at a distance. ${ }^{13}$ To enable these out-of-home helpers to play a more active and effective role, the CarePartner program is designed to automatically update a patient-designated support person regarding the patient's diabetes status based on the patient's 
IVR-reported information. The program also automatically provides as-needed problem-specific guidance on how to appropriately support the patients' efforts at self-management.

This study tested the hypothesis that patients with diabetes who choose to involve a support person in their automated telemonitoring program for self-management assistance demonstrate greater improvement in medication adherence (primary outcome) than patients who participate in the program without this component. Because psychological distress is often implicated in nonadherence, ${ }^{14}$ and because distressed individuals often attribute their difficulties to insufficient social support, ${ }^{15,16}$ we also examined whether distressed patients would benefit most from having this extra support.

\section{METHODS}

\section{Patient Eligibility and Recruitment}

Patient participants were recruited from 16 Department of Veterans Affairs (VA) outpatient clinics in Michigan, Illinois, Indiana, and Ohio between March 2010 and December 2012. Eligibility criteria were an ICD-9 diagnosis of type 2 diabetes, one or more primary care visits in the prior 12 months, and a current prescription for an antihyperglycemic medication. We excluded patients with diagnoses indicating cognitive impairment or severe mental illness and those who lived in a supervised residential facility. After we mailed potential participants an introductory letter, we screened them by telephone and obtained their written informed consent by mail. The study protocol was approved by human subjects committees at the Ann Arbor VA Healthcare System and University of Michigan.

\section{Baseline Assessment}

Research personnel conducted baseline assessments by telephone before patients began the IVR telemonitoring program. For the current analyses, we created sociodemographic indicators for minority race/ethnicity, married status, yearly income $<\$ 15,000$, and no education beyond high school. We measured functional health literacy with a screening index that demonstrated $80 \%$ sensitivity to the presence of inadequate health literacy in VA outpatients. ${ }^{17,18}$ Patients completed a checklist of common chronic conditions, which we classified based on distribution into tertile groups of $0-3,4-6$, and $>6$ comorbid conditions in addition to diabetes. From the Medical Outcomes Study 12-Item Short Form (SF-12), ${ }^{19}$ we computed the Physical Composite Summary (PCS; higher scores reflect better physical functioning) and Mental Composite Summary (MCS; higher scores reflect better psychological functioning and lower distress levels). Both the MCS and PCS have a potential range of 0 to 100 , with an age-corrected normative mean of $50(\mathrm{SD}= \pm 10)$. In lieu of an established MCS cutoff, we classified participants as distressed if their score fell at least one standard deviation below the population mean, i.e., MCS $\leq 40$. Finally, we measured long-term self-reported medication adherence with the well-validated Morisky Medication Adherence Scale (MMAS), on which higher scores reflect lower adherence. ${ }^{20,21}$ We used the developer's recommended cutoff of 2 to identify initially nonadherent patients for analysis, and we analyzed patient MMAS scores (i.e., continuous scores) at follow-up as a long-term measure of adherence during the intervention.

\section{Intervention}

Patients' IVR calls were scheduled weekly unless the patient was known to be hospitalized or had requested to stop calls for another reason (e.g., on vacation). Each week that a call was scheduled (i.e., call-week), the automated calling system made up to three call attempts on up to three patient-selected day/ time combinations. If a given week's call was incomplete after these nine attempts, the call was classified as incomplete, and the call data for that week were treated as missing. Calls typically lasted 5-7 minutes, and followed a tree-structured algorithm for presenting prerecorded queries to which patients could respond using their touch-tone keypad. Patients received tailored self-management messages based on their responses. The wording of questions and self-management educational messages was developed with input from experts in diabetes education, endocrinology, primary care, and automated telemonitoring service design. Further details of call content and flow can be obtained by contacting the corresponding author. Participating patients received the intervention for either three or six months. The difference in duration was due to our midproject design decision to extend the duration of participation in the program based upon initial results including positive patient feedback and indications that program benefits might continue accruing beyond three months. Data analyses were adjusted for three versus six months of participation.

Weekly IVR-Based Assessments. During the IVR calls, adherence to oral antihyperglycemic medication was assessed with the item, "Many people have difficulty taking their medicine exactly as prescribed by their doctor. How often would you say you took your diabetes medicine exactly as prescribed this past week?" The response scale ranged from 1 ("always") to 4 ("rarely or never"). For data analysis, we stringently dichotomized patients' weekly reported adherence as "always" taking medication exactly as prescribed versus anything less than perfect adherence, which tends to correct for inflated adherence self-reporting. ${ }^{22,23}$ In support of the validity of this method, we had previously found that the odds of nonadherence on this indicator doubled for each one-point increase in the MMAS total score. ${ }^{10}$ Additional IVR items assessed patients' general health in the past week and other diabetes parameters such as self-monitoring of blood glucose and inspecting one's feet for tissue damage.

Patient Self-Management Messages. As noted above, a diverse library of self-management support messages was 
developed by a multidisciplinary panel in order to ensure that the messages were engaging, clinically appropriate, easy to understand, and likely to be effective. When patients reported a difficulty in an IVR-assessed area, they heard a problem-specific self-management support message such as: If you sometimes forget to take your medicine, then consider buying a daily pill dispenser to help you keep track of when you should take your pills. If you already have a pill dispenser, then leave it out on your counter or table where you will see it often. Alternatively, when patients reported doing well, they heard a positive acknowledgement message such as: That's great. It's very important that you continue taking your diabetes medicine every day.

Clinician Notifications. When patients reported either significant medication nonadherence (i.e., adhering less than "most days") or a pattern of abnormal blood glucose or blood pressure levels, the system automatically faxed a notification to the patient's primary care team. Based upon clinician input, the thresholds for generating notifications were selected so as to have a low false-positive rate, provide actionable information, and efficiently use human resources for follow-up without overburdening clinicians with false alarms.

CarePartner Component. Patients could opt to designate a supportive family member or a close friend to be their "CarePartner" (CP). To participate as a CP, support persons needed to be $\geq 18$ years of age, have no history of cognitive or severe psychiatric impairment, and have access to e-mail. Additionally, they were required to be living outside the patient's residence, as our intention was that they would supplement any pre-existing care that the patient might already be receiving at home. We used the Norbeck Social Support Questionnaire (NSSQ) ${ }^{24}$ to help patients identify the individual best suited for this role. Before the program began, CPs underwent DVD-based training on providing diabetes self-management support and communicating effectively with the patient as well as with any of his or her pre-existing caregivers. After each IVR call that the patient completed, CPs were automatically emailed a structured report that summarized the patient's general health and self-management status. For areas in which the patient responded favorably, the report included suggestions on how the CP might reinforce this pattern. If the patient reported specific problems, the report explained the nature of those problems and provided detailed suggestions on ways to facilitate the patient's self-management. For example, [name] reported that she skipped taking her usual diabetes medication at least once during the week before the call. Although many diabetes patients skip their medication every now and then, this is not advised unless under the direction of a physician. Patients' reasons for skipping diabetes medication may include side effects, poor memory, running out of medication, and low blood sugar. Please contact [name] for more information about her use of diabetes medication, and any reasons for skipping it. You may be able to suggest a simple solution, such as setting up medication reminders, or urging her to refill her prescription. Other times, the reasons for skipping medication can be more complicated. These can include: coping with unpleasant side effects, staying motivated to prevent long-term complications, and depression.

\section{Post-Intervention Assessment}

After patients completed their three- or six-month telemonitoring intervention, research assessors re-administered the MMAS and the SF-12 by telephone, along with ancillary measures of patients' program satisfaction and related variables.

\section{Data Analysis}

Data were analyzed using Stata v.12.1. ${ }^{25}$ At the patient level, we computed descriptive statistics (frequency, mean, SD) for patient sociodemographic and clinical characteristics. The primary outcome variable was medication adherence, which was measured in two ways: long-term adherence as measured by the MMAS, and weekly adherence as measured by IVR. The first set of analyses tested for change in MMAS from baseline to post-intervention. Specifically, we conducted mixed-model linear regression analyses with restricted maximum likelihood estimation. These intent-to-treat analyses included all available observations, and missing post-intervention scores were imputed by carrying baseline scores forward. Initial models included trial arm (participated with $\mathrm{CP}$ [CP arm] versus participated alone [control arm]), baseline distress as measured by the MCS (distressed versus non-distressed), and time (baseline versus post-intervention), as well as their three-way and two-way interactions. Non-significant two-way interactions were removed from the model if the three-way interaction was removed due to non-significance. All main-effect terms were retained, regardless of their significance, in order to adjust final models. To investigate the process of change in patient adherence at the weekly level, we used logistic regression to analyze the factorial effects of distress, arm, and week on the probability of IVR-reported nonadherence. All available observations were analyzed, but missing week-level data were not imputed. All analyses were adjusted for program duration (three vs. six months) and control covariates identified through preliminary analyses presented below. When significant interactions between time and arm were identified, we performed stratified analyses in order to clarify the directionality and relative strength of the underlying associations. We also plotted the proportion of patients reporting nonadherence by time, and used locally weighted scatterplot smoothing (LOWESS $)^{26}$ to enhance the interpretability of the weekly data. We used a criterion of $p<0.05$ to evaluate statistical significance after adjusting confidence intervals for the clustering of repeated observations within patients. 


\section{RESULTS}

\section{Participant Characteristics}

Approximately $72 \%$ of eligible patients with diabetes who were initially contacted agreed to participate, and $33 \%$ of these 301 participants met the criteria for medication nonadherence, resulting in an analytic sample of 98 initially nonadherent patients. The typical participant (see Table 1) was a white male, as would be expected in VA settings. The mean age was $63( \pm 9.8)$ years, and $16 \%$ of patients were at least 70 years old. Two-thirds of participants were married or cohabitating, $25 \%$ had an annual household income of less than $\$ 15,000,44 \%$ had completed no education after high school, and half showed evidence of low functional health literacy. Seventy percent of participants had four or more chronic conditions in addition to diabetes. The patients' mean PCS score of 30.6 indicated that their physical functioning tended to be poor, while their mean baseline MCS score of 46.1 suggested that their psychological functioning tended to be mildly impaired, with $35 \%$ meeting study criteria for psychological distress. Approximately two-thirds (62\%) were enrolled into the longer (six-month) protocol.

\section{Potential Confounders}

As shown in Table 1, there were no significant baseline differences between participants enrolling with versus without a $\mathrm{CP}$, with the exception that patients who participated with a $\mathrm{CP}$ were more likely to screen positive for low functional health literacy compared to those who did not participate with a CP $(\mathrm{OR}=3.31,95 \% \mathrm{CI}: 1.43-7.66, p=0.005)$. There was a possible trend for patients in the $\mathrm{CP}$ arm to be relatively less likely to have a yearly income below $\$ 15,000$ ( $\mathrm{OR}=0.42$, $95 \%$ CI: $0.16-1.07, p=0.070)$. However, when analyzed together with low health literacy, the latter association weakened $(p=0.140)$ whereas the association with low health literacy persisted $(p=0.014)$. We therefore selected low functional health literacy for use as a control covariate.

\section{Call Completion and Attrition}

Patients participated in a total of 1,826 call-weeks, and completed an IVR call during 1,386 (76\%) of these. Likelihood of call completion was unrelated to patients' sociodemographic characteristics and number of medical comorbidities (all $p$ values $\geq 0.151$ ). However, call completion was more likely for patients in the CP arm (OR $=1.77,95 \% \mathrm{CI}: 1.01-3.09$, $p=0.046)$ and possibly less likely for those enrolled into the 6 month protocol (OR $=0.54,95 \%$ CI: $0.032-1.00, p=0.051)$. Dropout was defined as either an active request by a participant to withdraw from the study or completing less than $75 \%$ of scheduled calls. The rate of patient attrition was $19 \%$, and dropping out was likewise unrelated to sociodemographic variables or medical comorbidity (all $p$ values $\geq 0.132$ ), but was significantly less likely in the $\mathrm{CP}$ arm $(\mathrm{OR}=0.08,95 \%$ CI: $0.01-0.67, p=0.020$ ) and more likely among patients participating in the six-month protocol $(\mathrm{OR}=9.75,95 \% \mathrm{CI}$ : $1.22-77.99, p=0.032)$. Therefore, we also adjusted all primary analyses for study duration.

\section{Analysis of Change in Adherence Between Baseline and Post-Intervention}

As noted above, we evaluated whether adherence improved between baseline and post-intervention using mixed-model

Table 1. Patient Baseline Characteristics

\begin{tabular}{|c|c|c|c|c|}
\hline \multirow[b]{2}{*}{ Variable } & \multirow[b]{2}{*}{ Total sample $(n=303)$} & \multicolumn{3}{|c|}{ Participated with informal caregiver } \\
\hline & & No $(n=185)$ & Yes $(n=118)$ & $p$ value \\
\hline Age, mean $\pm \mathrm{SD}$ & $66.6 \pm 9.8$ & $66.5 \pm 8.9$ & $67.8 \pm 10.7$ & 0.426 \\
\hline Male, \% & 97.0 & 97.8 & 95.8 & 0.308 \\
\hline White, \% & 92.9 & 92.7 & 93.5 & 0.860 \\
\hline Married, \% & 67.3 & 70.3 & 62.8 & 0.171 \\
\hline Yearly household income & & & & 0.007 \\
\hline$<\$ 15,000$ & 26.1 & 20.5 & 34.8 & \\
\hline$\$ 15,000-\$ 29,000$ & 24.4 & 24.3 & 24.6 & \\
\hline$\$ 30,000-\$ 54,000$ & 28.7 & 31.9 & 23.7 & \\
\hline$>\$ 55,000$ & 20.8 & 23.2 & 17.0 & \\
\hline Education level, some college or higher, $\%$ & 53.0 & 50.0 & 57.6 & 0.195 \\
\hline Employed, \% & 18.2 & 17.2 & 19.8 & 0.571 \\
\hline Comorbid conditions, ${ }^{\mathrm{a}}$ mean $\pm \mathrm{SD}$ & $4.8 \pm 2.2$ & $4.7 \pm 2.1$ & $5.0 \pm 2.2$ & 0.360 \\
\hline Hypertension diagnosis, \% & 85.7 & 84.7 & 87.3 & 0.531 \\
\hline Inadequate healthcare literacy, \% & 52.2 & 44.3 & 64.4 & 0.001 \\
\hline $\mathrm{PCS},{ }^{\mathrm{b}}$ mean $\pm \mathrm{SD}$ & $32.3 \pm 12.2$ & $32.1 \pm 12.1$ & $32.6 \pm 12.4$ & 0.746 \\
\hline $\mathrm{MCS},{ }^{\mathrm{c}}$ mean $\pm \mathrm{SD}$ & $50.0 \pm 11.7$ & $49.3 \pm 12.0$ & $51.0 \pm 11.2$ & 0.230 \\
\hline MMAS, ${ }^{\mathrm{d}}$ mean $\pm \mathrm{SD}$ & $1.20 \pm 0.96$ & $1.17 \pm 0.99$ & $1.26 \pm 0.89$ & 0.456 \\
\hline Elevated MMAS, \% & 35.6 & 33.5 & 39.0 & 0.332 \\
\hline
\end{tabular}

${ }^{a}$ Based on self-reported hypertension, cardiovascular disease, hyperlipidemia, cancer, stroke, arthritis, chronic lung disease, migraine, asthma, or low back pain

${ }^{b}$ Physical Composite Summary of the Medical Outcomes Study 12-Item Short Form. Higher scores indicate better functioning.

${ }^{c}$ Mental Composite Summary of the Medical Outcomes Study 12-Item Short Form. Higher scores indicate better functioning.

${ }^{d}$ Morisky Medication Adherence Scale, phrased for diabetes medication. Higher scores indicate greater medication nonadherence. 
regression to analyze the effects of arm, distress, and time upon MMAS scores after adjusting for low functional health literacy and study duration. In the initial full model (results not shown), the three-way interaction term (arm X distress X time) was not statistically significant ( $p=0.809$ ), and therefore was dropped from the model, along with the arm X distress interaction term ( $p=0.844)$. The distress $\mathrm{X}$ time interaction fell short of statistical significance $(b=0.37,95 \% \mathrm{CI}:-0.75-0.02$, $p<0.062$ ), and was also dropped from the model.

In the final trimmed model (see Table 2), there was a general tendency for MMAS scores to decrease (i.e., better adherence) between baseline and post-intervention $(b=-0.58,95 \% \mathrm{CI}$ : $-0.82--0.33, p<0.001)$. Importantly, there was a significant arm X time interaction $(b=-0.60,95 \% \mathrm{CI}$ : 0.98-0.23, $p=0.001$ ), indicating greater improvement in MMAS scores for the CP arm (see Fig. 1). Follow-up arm-stratified analyses (results not shown) indicated that the regression slope estimating improvement in MMAS scores over time for the CP $\operatorname{arm}(b=-1.18,95 \% \mathrm{CI}:-1.43--0.91, p<0.001)$ was significantly steeper $\left(t_{(95)}=3.32, p=0.001\right)$ than that for the control arm $(b=-0.57,95 \% \mathrm{CI}:-0.83--0.32, p<0.001)$.

\section{Analysis of Changes in Weekly Nonadherence}

The logistic analysis of weekly medication nonadherence indicated a significant three-way interaction among arm, time, and baseline distress $(\mathrm{AOR}=0.72,95 \% \mathrm{CI}$ : 0.56-0.93, $p=0.010$, see Table 3 ). This was due to the presence of a significant arm $\mathrm{X}$ week interaction for the distressed group (AOR $=0.72,95 \%$ CI: 0.55-0.93, $p=0.013$, see Table 3 ) but not for the non-distressed group $(\mathrm{AOR}=1.04,95 \% \mathrm{CI}$ : $0.97-$ $1.11, p=0.246$, results not shown). Specifically, the odds of nonadherence tended to decrease $25 \%$ per week for distressed patients in the CP arm (AOR $=0.75,95 \%$ CI: 0.58-0.97, $p=0.030)$, but remained constant across weeks for distressed patients in the control arm $(\mathrm{AOR}=1.01,95 \% \mathrm{CI}$ : 0.94-1.08, $p=0.820$ ). This pattern is evident in Fig. 2, which displays the probability of nonadherence over time among distressed patients separately for patients with versus without a CP.

Table 2. Results of Mixed-Model Regression Analysis of Change from Baseline to Post-Intervention ( $n=98$ patients assessed at two time points)

\begin{tabular}{lcrrr}
\hline \hline $\begin{array}{l}\text { Outcome } \\
\text { variable }\end{array}$ & Predictor variable & \multicolumn{1}{l}{$\boldsymbol{b}^{\boldsymbol{a}}$} & $\mathbf{9 5} \% \mathbf{C I}$ & $\boldsymbol{p}$ value \\
\hline Adherence $^{\mathrm{b}}$ & Low health literacy & -0.11 & $-0.39-0.17$ & 0.444 \\
Study duration $^{\text {Distress }}$ & 0.04 & $-0.32-0.40$ & 0.820 \\
Arm $^{\mathrm{d}}$ & 0.15 & $-0.11-0.40$ & 0.259 \\
Time $^{\mathrm{e}}$ & -0.02 & $-0.40-0.35$ & 0.904 \\
Arm X Time & -0.58 & $-0.82--0.33$ & $<0.001$ \\
\hline
\end{tabular}

${ }^{a}$ Raw regression (beta) coefficient

${ }^{b}$ As measured by the Morisky Medication Adherence Scale (MMAS); higher scores indicate lower adherence

${ }^{c}$ Distressed group coded as 1

${ }^{d} \mathrm{CP}$ arm coded as 1

${ }^{e}$ Post-intervention (coded as 1) versus baseline

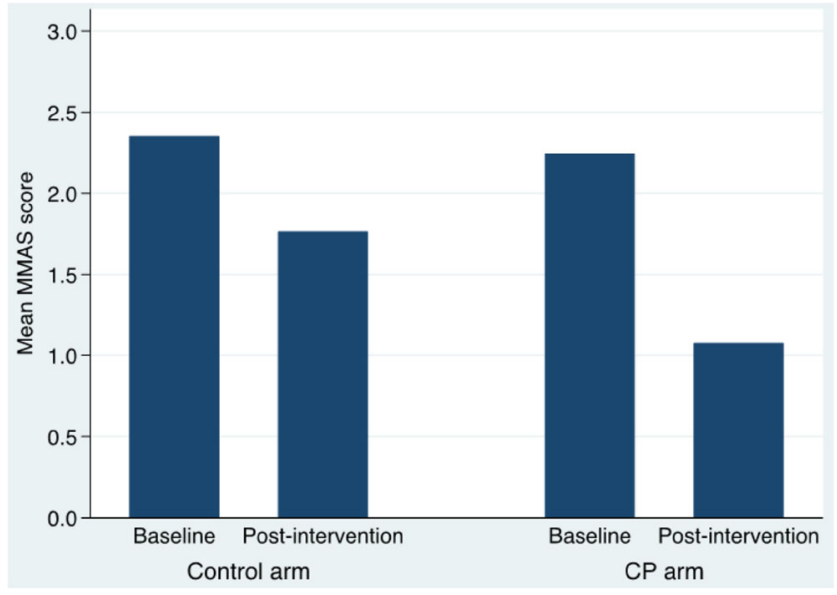

Figure 1 Mean Morisky Medication Adherence Scale (MMAS) scores by arm and time.

Because preliminary analyses indicated that patients in the CP arm were more likely to complete their IVR calls, we repeated the analysis after further adjusting for proportion of calls completed. Likewise, we repeated the analysis when excluding patients who received only three months of intervention in order to check whether intended program duration influenced the findings. However, in both auxiliary analyses, the pattern of significant effects suggesting substantially better adherence for distressed patients who participated with a $\mathrm{CP}$ remained unchanged.

\section{DISCUSSION}

While the benefits of standard telemonitoring and selfmanagement support for chronically ill patients are well documented, ${ }^{27}$ to our knowledge, the CarePartner program is the only such intervention that allows patients to participate with a patient-selected support person who receives regular updates

Table 3 Logistic Regression Models Predicting Weekly IVRReported Medication Nonadherence ${ }^{\mathrm{a}}$

\begin{tabular}{lccr}
\hline \hline Predictor variable & AOR $^{\mathbf{b}}$ & $\mathbf{9 5} \% \mathbf{C I}$ & $\boldsymbol{p}$ value \\
\hline Full model ( $n=1,386$ calls with 98 patients) & & \\
Low health literacy & 0.50 & $0.20-1.30$ & 0.156 \\
Study duration & 0.44 & $0.13-1.48$ & 0.184 \\
Distress $^{\mathrm{d}}$ & 0.96 & $0.37-2.51$ & 0.933 \\
Arm & 0.72 & $0.24-2.18$ & 0.562 \\
Week & 0.96 & $0.91-1.00$ & 0.077 \\
Distress X Week & 1.01 & $0.99-1.15$ & 0.082 \\
Arm X Week & 1.03 & $0.96-1.11$ & 0.385 \\
Distress X Arm & 1.64 & $0.29-9.41$ & 0.576 \\
Distress X Arm X Week & 0.72 & $0.56-0.93$ & 0.010 \\
Follow-up model, distressed group $(n=450$ & calls with 33 patients) \\
Low health literacy & 0.09 & $0.03-0.29$ & $<0.001$ \\
Study duration & 0.79 & $0.11-5.81$ & 0.814 \\
Arm & 2.24 & $0.24-20.95$ & 0.480 \\
Week & 1.02 & $0.96-1.08$ & 0.629 \\
Arm X Week & 0.72 & $0.55-0.93$ & 0.013 \\
\hline
\end{tabular}

${ }^{a}$ Nonadherence coded as 1 , for consistency with MMAS

${ }^{b}$ Adjusted odds ratio

${ }^{c}$ Six-month study duration coded as 1

${ }^{d}$ Distressed group coded as 1

${ }^{e} \mathrm{CP}$ group coded as 1 


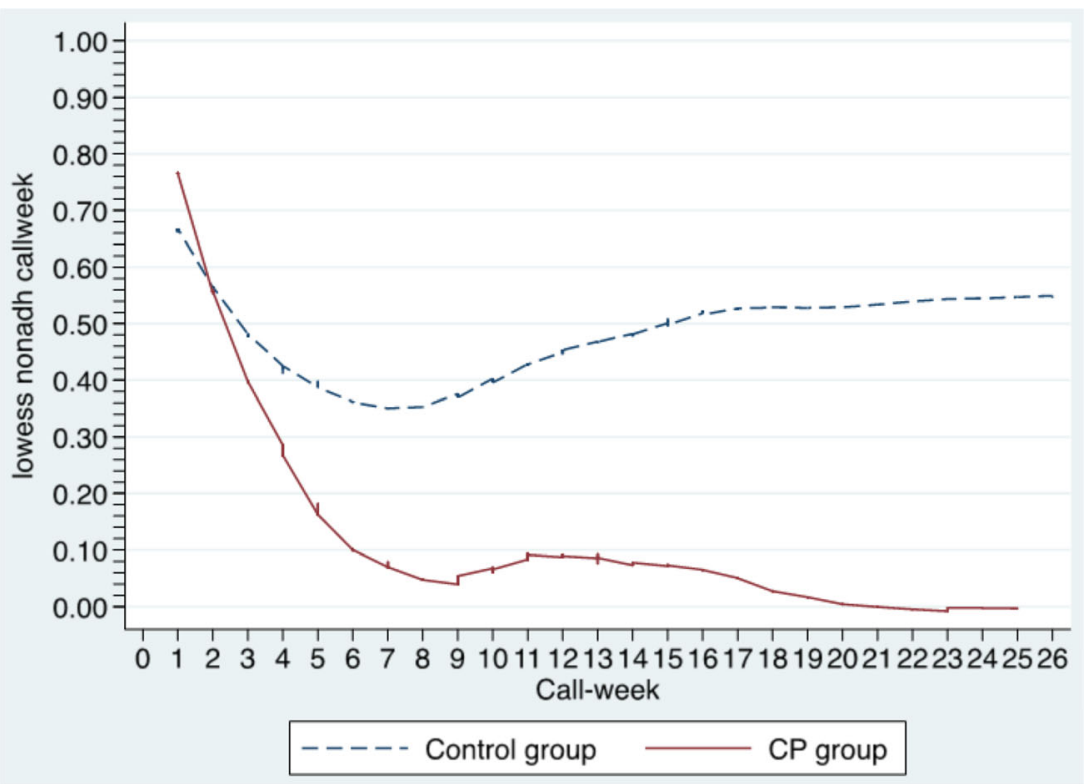

Figure 2 Probability of nonadherence by arm and call-week.

about the patient's status and guidance about how they can help. In this study, we found that patients who availed themselves of this option showed significantly greater gains in long-term adherence to antihyperglycemic medication compared to those who participated in the program without a partner. Analyses of patient IVR-reported weekly medication adherence indicated that this advantage occurred specifically among patients who were psychologically distressed at enrollment.

It is important to emphasize that these benefits were observed above and beyond the benefits of a fairly active control condition. Furthermore, although all participants, by definition, were initially nonadherent, attrition and incomplete calls were not only surprisingly uncommon (at $19 \%$ and $24 \%$, respectively) but also unrelated to patient sociodemographic characteristics. While the non-randomized design does not allow us to draw definitive causal conclusions, these encouraging findings strongly suggest that properly structured selfmanagement support from a friend or out-of-home family member has the potential to boost adherence outcomes for some of the most vulnerable patient groups.

A variety of factors could conceivably have influenced whether patients opted to participate along with a support person. By definition, CarePartner participation required that the patient had a pre-existing relationship with a supportive individual who was willing to review ongoing feedback on the patient's status and act helpfully upon this information. Not surprisingly, the majority of CarePartners were an adult son or daughter of the patient, and therefore likely already well invested in the patient's well-being. Typically two to three decades younger than the patient, these individuals were also quite likely to be regular users of e-mail and text messaging, and therefore probably comfortable with the program's communication modalities. A second likely determinant of patient choice of whether to include a CP was the patient's preference for privacy and autonomy versus social connectedness and support. Patients choosing the CarePartner option also tended to be challenged by low health literacy and possibly low income. Because strong social ties are linked to better health, ${ }^{28}$ this raises the interesting possibility that socioeconomically disadvantaged patients might select this option in an active effort to mitigate their risk factors. Stated simply, intensified support may appeal to those who need it most.

Although this study was not designed to determine how or why having a support person might increase medication adherence, several mediating mechanisms are possible. Perhaps the three most plausible of these are (a) emotional support leading to improved ability to regulate one's own behavior, (b) direct assistance with diabetes problem-solving provided by CPs, and (c) CP reinforcement of adherence. First, we believe that distressed patients, in particular, may desire and benefit from increased support, as they tend to attribute their difficulties to insufficient support. ${ }^{16}$ To the extent that patients' difficulties with adherence are rooted in their general distress, increased contact with a trusted helper may improve patients' affective state and motivation to care for themselves. Second, we encouraged patients and their support persons to engage in collaborative problem-solving to identify effective strategies for addressing barriers to medication adherence, a strategy reminiscent of the common adage, "two heads are better than one." Finally, social reinforcement likely enhanced outcomes among patients with a CarePartner, as praise from a supportive individual is a potent reinforcer of health behavior. ${ }^{29}$ On a more concrete level, simply receiving "live" support may help to counteract the potentially impersonal or repetitive nature of automated IVR calls. 
The temporal patterns of weekly nonadherence (see Fig. 2) suggest some interesting implications. Although we had no specific a priori expectations for the shape of these trajectories, improvement was initially rapid, with virtually all net gains occurring during the first six to eight weeks of the program. After this point, the probability of nonadherence in the $\mathrm{CP}$ arm eventually approached zero. In contrast, the control group demonstrated a relatively weak initial response, followed by a gradual but nearly complete return to baseline levels, possibly reflecting a Hawthorne effect. Further research should explore whether poor initial improvement predicts a poor outcome. At the opposite end of the study timeline, it would also be interesting to determine whether improved adherence is maintained beyond the six-month point.

\section{Limitations}

This study had several limitations. First and foremost, participation with a support person was determined by patient preference rather than random assignment. Therefore, we cannot rule out that some unmeasured factor influenced the observed effects. On the other hand, preference trials usually have better external validity than randomized controlled trials because they more closely represent the conditions of usual clinical care. Additionally, while both distress and low health literacy increase the risk of poor diabetes selfmanagement and hyperglycemia, ${ }^{30,31}$ our statistical adjustments for these factors probably helped reduce potential confounders.

A second potential limitation was our reliance on selfreported adherence, although our stringent adherence cutoff and brief reporting interval may have counteracted the potential biases usually associated with self-reported adherence. ${ }^{22}$ While patients may have been reluctant to admit difficulties if they were uncomfortable with the automated interface of the IVR calls, IVR-obtained health data are at least as reliable as those obtained using structured interview or review of medical records. ${ }^{7,32}$ Notwithstanding this fact, follow-up studies should include additional data sources, such as electronic event monitoring, medication refills, or bioassays, in order to validate impacts on patient adherence. A third significant issue was the limited sample size, which may have adversely affected our sensitivity to other effects. Although adherence is a powerful predictor of glycemic control, ${ }^{2}$ further research is needed to determine whether having a support person also improves medical outcomes. Given the unique demographics of the VA setting, replication studies are needed in order to generalize the findings to other populations. Finally "psychological distress" is a heterogeneous construct. Insofar as adherence has multiple determinants in distressed medical patients, ${ }^{33,34}$ there may be clinically important subgroups within this generalized category who may respond differently to involving a support person in their telemonitoring-based self-management program.

\section{CONCLUSIONS}

Patients with diabetes who are both nonadherent and psychologically distressed are often considered among the most challenging groups to treat, yet we found that they engaged readily and persistently with automated telehealth support. Our key finding was that medication adherence improved only among those who chose to incorporate a support person into their telehealth program. It is important to emphasize that these benefits were observed over and above those of a fairly active control condition. If confirmed in an ongoing randomized controlled trial, these findings may benefit large numbers of patients with diabetes who might otherwise lack self-management support.

The findings also raise the intriguing possibility that intensified support might benefit patients with other chronic conditions that have a major self-management component. If so, there may be numerous opportunities to creatively adapt this strategy across chronic illnesses, settings, and delivery modalities. Accordingly, we are currently evaluating similar programs for patients with congestive heart failure, depression, and other conditions. If this patient-centered strategy can demonstrate generalizable benefits, it may help to reduce the long-standing gap between what many patients need and what most healthcare settings can realistically provide.

Acknowledgments: JDP is a VA Senior Research Career Scientist. RT is supported by a VA Career Development Award (grant \# CDA-09206). Other financial support was provided by the Michigan Diabetes Translational Research Center (grant P30DK092926) and grant R18DK088294 from the National Institute of Diabetes and Digestive and Kidney Diseases. The views expressed in this manuscript are those of the authors and do not necessarily represent the opinions of the Department of Veterans Affairs. We appreciate the helpful statistical consultation provided by Ananda Sen, $\mathrm{PhD}$, at the University of Michigan.

Conflict of Interest: The authors each declare that they have no conflict of interest.

Corresponding Author: James E. Aikens, PhD; Department of Family Medicine, ?University of Michigan, 1018 Fuller Street, Ann Arbor, MI 48104-1213, USA (e-mail: aikensj@umich.edu).

\section{REFERENCES}

1. Alberti KG, Zimmet PZ. Definition, diagnosis and classification of diabetes mellitus and its complications. Part 1: diagnosis and classification of diabetes mellitus. Provisional report of a WHO consultation. Diabet Med. 1998;15:539-553.

2. Aikens JE, Piette JD. Longitudinal association between medication adherence and glycaemic control in type 2 diabetes. Diabet Med. 2013;30:338-344.

3. Anderson RM, Funnell MM, Aikens JE, et al. Evaluating the efficacy of an empowerment-based self-management consultant intervention: results of a two-year randomized controlled trial. Ther Patient Educ. 2009;1:3-11.

4. Piette JD. Moving diabetes management from clinic to community: development of a prototype based on automated voice messaging. Diabetes Educ. 1997;23:672-680.

5. Schwartz S. What works. IVR system helps diabetes center triple patient load; increases care level. Health Manag Technol. 1998;19:41.

6. Piette JD. Interactive behavior change technology to support diabetes selfmanagement: where do we stand? Diabetes Care. 2007;30:2425-2432. 
7. Piette JD, Rosland AM, Marinec NS, Striplin D, Bernstein SJ, Silveira MJ. Engagement with automated patient monitoring and self-management support calls: experience with a thousand chronically-ill patients. Med Care. 2013;51:216-223.

8. Piette JD, Weinberger M, McPhee SJ, Mah CA, Kraemer FB, Crapo LM Do automated calls with nurse follow-up improve self-care and glycemic control among vulnerable patients with diabetes? American J Med. 2000; 108:20-27.

9. Aikens JE, Rosland AM, Piette JD. Improvements in illness selfmanagement and psychological distress associated with telemonitoring support for adults with diabetes. Prim Care Diabetes. In press.

10. Aikens JE, Zivin K, Trivedi R, Piette JD. Diabetes self-management support using mHealth and enhanced informal caregiving. J Diabetes Complications. 2014;28:171-176.

11. Armour T, Norris S, Jack L, Zhang X, Fisher L. The effectiveness of family interventions in people with diabetes mellitus: a systematic review. Diabet Med. 2005;22:1295-1305.

12. Rosland A, Heisler M, Janevic MR, Connell CM, Langa KM, Kerr EA, Piette JD. Current and potential support for chronic disease management in the United States: The perspective of family and friends of chronically ill adults. Fam Syst Health. 2013;31:119-131.

13. Piette JD, Rosland AM, Silveira M, Kabeto M, Langa KM. The case for involving adult children outside of the household in the self-management support of older adults with chronic illnesses. Chronic Illn. 2010;6:34-45.

14. Gonzalez JS, Peyrot M, McCarl LA, et al. Depression and diabetes treatment nonadherence: a meta-analysis. Diabetes Care. 2008;31:2398-2403.

15. Griffith J. Social support providers: Who are they? Where are they met? and the relationship of network characteristics to psychological distress. Basic Appl Soc Psychol. 1985;6:41-60.

16. Strine $\mathbf{T W}$, Kroenke $\mathbf{K}$, Dhingra $\mathbf{S}$, et al. The associations between depression, health-related quality of life, social support, life satisfaction, and disability in community-dwelling US adults. J Nerv Ment Dis. 2009;197:61-64.

17. Chew LD, Bradley KA, Boyko EJ. Brief questions to identify patients with inadequate health literacy. Fam Med. 2004;36:588-594.

18. Chew LD, Grill JP, Snyder A, Bradley KA, Nugent SM, Baines AD. Validation of screening questions for limited health literacy in a large VA outpatient population. J Gen Intern Med. 2008;23:561-566.

19. Ware J Jr, Kosinski M, Keller SD. A 12-item short-form health survey: construction of scales and preliminary tests of reliability and validity. Med Care. 1996;34:220-233.
20. Morisky DE, Green LW, Levine DM. Concurrent and predictive validity of a self-reported measure of medication adherence. Med Care. 1986;24:67-74.

21. Morisky DE, Ang A, Krousel-Wood M, Ward HJ. Predictive validity of a medication adherence measure in an outpatient setting. $\mathrm{J}$ Clin Hypertens (Greenwich). 2008;10:348-354.

22. Stirratt M, Dunbar-Jacob J, Crane H, et al. Self-report measures of medication adherence behavior: recommendations on optimal use. Manuscript under review 2014.

23. Garber MC, Nau DP, Erickson SR, Aikens JE, Lawrence JB. The concordance of self-report with other measures of medication adherence: a summary of the literature. Med Care. 2004;42:649-652.

24. Attkisson CC, Zwick R. The client satisfaction questionnaire. Psychometric properties and correlations with service utilization and psychotherapy outcome. Eval Program Plann. 1982;5:233-237.

25. StataCorp. Stata Statistical Software: Release 13.1. College Station, TX: StataCorp LP; 2014.

26. Cleveland wS. Robust locally weighted regression and smoothing scatterplots. J Am Stat Assoc. 1979;74:829-836.

27. Paré G, Jaana M, Sicotte C. Systematic review of home telemonitoring for chronic diseases: the evidence base. J Am Med Inform Assoc. 2007; 14:269-277.

28. Berkman LF. Social ties and mental health. J Urban Health. 2001;78:458-467.

29. Perry CL, Baranowski T, Parcel GS. How individuals, environments, and health behavior interact: Social learning theory. In: Glanz K, Lewis FM, Rimer B, eds. Health Behavior and Health Education. San Francisco: Jossey-Bass; 1990:161-186.

30. Aikens JE, Piette JD. Diabetic patients' medication underuse, illness outcomes, and beliefs about antihyperglycemic and antihypertensive treatments. Diabetes Care. 2009;32:19-24.

31. Aikens JE. Prospective associations between emotional distress and poor outcomes in type 2 diabetes. Diabetes Care. 2012;35:2472-2478.

32. Piette JD. Interactive voice response systems in the diagnosis and management of chronic disease. Am Manag Care. 2000;6:817-827.

33. Aikens JE, Nease DE, Klinkman MS. Explaining patients' beliefs about the necessity and harmfulness of antidepressants. Ann Fam Med. 2008:6:23-29.

34. Aikens JE, Nease DE Jr, Nau DP, Klinkman MS, Schwenk TL. Adherence to maintenance-phase antidepressant medication as a function of patient beliefs about medication. Ann Fam Med. 2005;3:23-30. 